\title{
LETTER \\ Carrier Frequency Synchronization for OFDM Systems in the Presence of Phase Noise
}

\author{
Yong-Hwa KIM ${ }^{\dagger \mathrm{a})}$, Jong-Ho LEE ${ }^{\dagger}$, Nonmembers, and Seong-Cheol $\mathrm{KIM}^{\dagger}$, Member
}

SUMMARY A carrier frequency synchronization scheme is proposed for orthogonal frequency-division multiplexing (OFDM) systems in the presence of phase noise (PHN). In the proposed scheme, carrier frequency synchronization is performed based on the maximum-likelihood (ML) algorithm using an OFDM preamble symbol. The proposed scheme is compared with conventional methods. Simulation results are presented to illustrate the effectiveness of the proposed scheme in the presence of PHN.

key words: orthogonal frequency-division multiplexing (OFDM), carrier frequency offset (CFO), channel estimation, phase noise (PHN)

\section{Introduction}

Orthogonal frequency-division multiplexing (OFDM) has recently received considerable attention for its robustness against frequency selective channels. It is known that the drawback of the OFDM system is the sensitivity of the receiver to oscillator instabilities, such as carrier frequency offset (CFO) and phase noise (PHN). Moreover, the coherent detection of OFDM signals requires channel estimation to mitigate amplitude and phase distortions in a fading channel.

A number of channel impulse response (CIR) estimation algorithms [1], [2] and CFO estimation algorithms [3][5] have been proposed in the literature. The CIR is estimated assuming zero CFO or CFO is estimated assuming perfect channel estimation. Joint frequency offset and CIR estimation issue is also highlighted in the papers [6]-[8]. In [6], an adaptive approach (i.e. steepest descent algorithm) was employed to avoid the complexity of joint maximumlikelihood (ML) estimation.

However, PHN impairs the joint CFO and CIR estimation. Two approaches to modeling $\mathrm{PHN}$ as a random process have been investigated in the literature. One can be obtained by measuring a real tuner with a phase-locked loop (PLL) [9]-[11], [15]. The other does the Wiener PHN model of a free-running oscillator [10], [12]. We choose the former model. A PLL is basically an oscillator whose frequency is locked onto some frequency component of the reference signal. This is done with a feedback control loop as shown in Fig. 1. The phase of a local voltage-controlled oscillator (VCO) is compared with the phase of a low-noise reference signal. The difference of the two phases is low-pass

\footnotetext{
Manuscript received September 29, 2006.

Manuscript revised November 9, 2006.

${ }^{\dagger}$ The authors are with Institute of New Media and Communications, School of Electrical Engineering and Computer Science, Seoul National University, Seoul, Korea.

a) E-mail: yhkim@maxwell.snu.ac.kr

DOI: 10.1093/ietcom/e90-b.6.1543
}

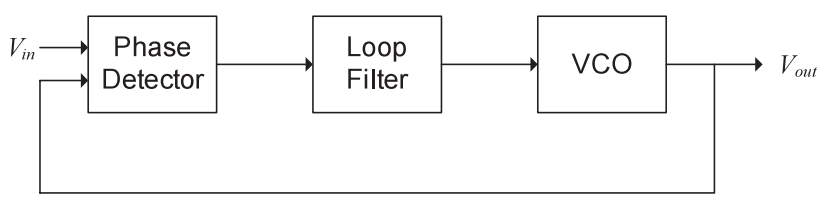

Fig. 1 Basic PLL block diagram.

filtered by loop filter and applied to the controlling node of the VCO.

In this letter, we introduce a system model with CFO, PHN and unknown but deterministic CIR. Considering this model, we propose a carrier frequency synchronization scheme based on the ML algorithm employing an OFDM preamble symbol to perform the fine frequency synchronization. In the proposed scheme, the least-square (LS) channel estimate results, which is based on the cyclic-prefix (CP)-based CFO estimation and compensation, are used to obtain the fine $\mathrm{CFO}$ estimate.

\section{System Model}

Let $N$ be the number of sub-carriers, $N_{C P}$ be the number of $\mathrm{CP}$ and $P+1$ be the number of modulated sub-carriers. Note that $N-P-1$ sub-carriers at the edges of the spectrum (i.e. virtual sub-carriers) are not used and the modulated sub-carriers can be indexed by the numbers from $-P / 2$ to $P / 2$. The time-domain OFDM symbol is generated by $N$ point IDFT and CP is appended at the beginning of each time-domain OFDM symbol.

The time-domain received signal vector $\boldsymbol{r}=[r(0) r(1)$ $\cdots r(N-1)]^{T}$ over a frequency selective fading channel, after $\mathrm{CP}$ removal, can be expressed as

$$
\boldsymbol{r}=\boldsymbol{\Omega}_{v} \boldsymbol{F}_{\phi} \boldsymbol{F} \boldsymbol{A} \boldsymbol{H}+\boldsymbol{w}
$$

where $\boldsymbol{A}=\operatorname{diag}\{a(-P / 2), \cdots, a(0), \cdots, a(P / 2)\}$ denotes a diagonal transmitted symbol matrix in the preamble and $[\boldsymbol{F}]_{n, p}=\frac{1}{\sqrt{N}} \exp \left(j \frac{2 \pi p}{N} n\right)$ where $0 \leq n<N$ and $|p| \leq P / 2$. Phase noise (PHN) and a carrier frequency offset (CFO) $v$ are presented in the matrix $\boldsymbol{F}_{\phi}$ and $\boldsymbol{\Omega}_{v}$, respectively, given by

$$
\begin{aligned}
& \boldsymbol{F}_{\phi}=\operatorname{diag}\left\{\exp \left(j \phi_{0}\right), \exp \left(j \phi_{1}\right), \cdots, \exp \left(j \phi_{N-1}\right)\right\} \\
& \boldsymbol{\Omega}_{v}=\operatorname{diag}\left\{\Omega_{v}(0), \Omega_{v}(1), \cdots, \Omega_{v}(N-1)\right\}
\end{aligned}
$$

and

$$
\Omega_{v}(n)=\exp \left(j \frac{2 \pi v}{N} n\right)
$$


where $\phi_{n}$ is the sample of the PHN process $\phi(t)$ generated by the receiver oscillator with a PLL. Assuming ideal timing synchronization and sufficient $\mathrm{CP}$, the channel frequency response $\boldsymbol{H}$ can be given as $\boldsymbol{D} \boldsymbol{h}$ where $\boldsymbol{h}$ $=[h(0) h(1) \cdots h(L-1)]^{T}$ denotes the channel impulse response $(\mathrm{CIR})$ with $L$ multi-paths and $[\boldsymbol{D}]_{p, l}=\exp \left(-j \frac{2 \pi p}{N} l\right)$ where $0 \leq l<L$. Moreover, the additive white Gaussian noise vector $\boldsymbol{w}=[w(0) w(1) \cdots w(N-1)]^{T}$ has the covariance matrix of $\sigma_{w}^{2} \boldsymbol{I}_{N}$ where $\boldsymbol{I}_{N}$ denotes an $N \times N$ identity matrix.

We consider a PHN process model as a zero-mean, stationary and finite-power random process, which can be obtained by measuring a real tuner with a PLL [9]-[11], [15]. The output of the VCO with PHN can be represented as $x_{c}(t)$ $=e^{j\left(2 \pi f_{c} t+\phi(t)\right)}$ where $f_{c}$ is the desired frequency of the reference signal. The autocorrelation function of $x_{c}(t)$ can be calculated as

$$
\begin{aligned}
R_{x_{c}}(t, \tau) & =E\left[x_{c}(t) x_{c}^{*}(t+\tau)\right] \\
& =e^{-j 2 \pi f_{c} \tau} E\left[e^{-j(\phi(t+\tau)-\phi(t))}\right] .
\end{aligned}
$$

Since the PHN process $\phi(t)$ is asymptotically a zero mean Gaussian process [15], the difference $\phi(t+\tau)-\phi(t)$ in (5) is a zero-mean Gaussian process with variance equal to $\sigma_{\phi}^{2}(t, \tau)$ $=E\left[|\phi(t+\tau)-\phi(t)|^{2}\right]$. Thus,

$$
R_{x_{c}}(t, \tau)=e^{-j 2 \pi f_{c} \tau} e^{-(1 / 2) \sigma_{\phi}^{2}(t, \tau)} .
$$

Since $\phi(t)$ is modeled as a stationary random process [15], $\sigma_{\phi}^{2}(t, \tau)=\sigma_{\phi}^{2}(\tau)$ can be calculated as

$$
\sigma_{\phi}^{2}(\tau)=2 R_{\phi}(0)-2 R_{\phi}(\tau)
$$

where $R_{\phi}(\tau)=E[\phi(t) \phi(t+\tau)]$ is an autocorrelation function of $\phi(t)$. Since $R_{\phi}(\tau)$ is sufficiently small to approximated by $e^{R_{\phi}(\tau)} \approx 1+R_{\phi}(\tau)$ [11], [15], (6) can be approximated as

$$
R_{x_{c}}(t, \tau) \approx e^{-j 2 \pi f_{c} \tau} e^{-R_{\phi}(0)}\left(1+R_{\phi}(\tau)\right) .
$$

The Fourier transform of (8), which is the power spectral density (PSD) of the output, is given by

$$
S_{x_{c}}(f)=e^{-R_{\phi}(0)}\left(\delta\left(f+f_{c}\right)+S_{\phi}\left(f+f_{c}\right)\right)
$$

where $\delta(\cdot)$ is the Dirac delta function and $S_{\phi}(f)$ is the PSD of $\phi(t)$. The PSD of the PLL output may be calculated as the specifications of the PLL [11], [15] or be directly measured by a spectrum analyzer [9], [11], [15]. Using (8), (9) and the PSD of the PLL output, $S_{\phi}(f)$ and $R_{\phi}(\tau)$ can be obtained. The sampled PHN process $\varphi=\left[\phi_{0} \phi_{1} \cdots \phi_{N-1}\right]^{T}$ can be modeled as a zero mean Gaussian vector of the covariance matrix $\boldsymbol{\Phi}$, which is given by

$$
[\boldsymbol{\Phi}]_{i, j}=R_{\phi}\left(|i-j| \frac{T}{N}\right)
$$

where $T$ is the OFDM symbol period.

Assuming that PHN is sufficiently small to approximate $\exp \left(j \phi_{n}\right) \approx 1+j \phi_{n}$ by Taylor series expansion [11], (1) can be expressed as

$$
\boldsymbol{r} \approx \boldsymbol{\Omega}_{v} \boldsymbol{F} \boldsymbol{A} \boldsymbol{D h}+\operatorname{diag}\left\{j \boldsymbol{\Omega}_{v} \boldsymbol{F} \boldsymbol{A D h}\right\} \boldsymbol{\phi}+\boldsymbol{w} .
$$

Here, we define the PHN-plus-noise as $\boldsymbol{V}=\operatorname{diag}\left\{j \boldsymbol{\Omega}_{v}\right.$ $\boldsymbol{F} \boldsymbol{A D h}\} \boldsymbol{\phi}+\boldsymbol{w}$. Since $\boldsymbol{\varphi}$ and $\boldsymbol{w}$ are independent and zero mean Gaussian distributed, $\boldsymbol{V}$ is also zero mean Gaussian with the covariance matrix $\boldsymbol{\Sigma}_{\boldsymbol{h}, v}=$ $\operatorname{diag}\left\{\boldsymbol{\Omega}_{v} \boldsymbol{F} \boldsymbol{A} \boldsymbol{D} \boldsymbol{h}\right\} \boldsymbol{\Phi} \operatorname{diag}\left\{\boldsymbol{\Omega}_{v} \boldsymbol{F} \boldsymbol{A} \boldsymbol{D} \boldsymbol{h}\right\}^{H}+\sigma_{w}^{2} \boldsymbol{I}_{N}$.

\section{Carrier Frequency Synchronization}

The coarse CFO estimate $v_{c}$ can be obtained using the redundancy generated by the $\mathrm{CP}$ [3]. Using the coarse CFO $v_{c}$, the LS CIR estimate $\hat{\boldsymbol{h}}$ can be obtained by

$$
\hat{\boldsymbol{h}}=\left(\boldsymbol{S}^{H} \boldsymbol{S}\right)^{-1} \boldsymbol{S}^{H} \mathbf{\Omega}_{v_{c}}^{H} \boldsymbol{r}
$$

where $\boldsymbol{S}=\boldsymbol{F} \boldsymbol{A} \boldsymbol{D}$ and $(\cdot)^{H}$ denotes conjugated transpose. Note that the CIR length $L$ should be known in order to construct $S$ in (12). So the additional algorithm for the CIR length estimation is needed. However, we find that the estimator is robust to the overestimated CIR length. So the CIR length $L$ can be simply replaced by $N_{C P}$ under the sufficient CP. Here, we assume that the PHN covariance matrix $\boldsymbol{\Phi}$ is known. Furthermore, using the LS CIR $\hat{\boldsymbol{h}}$, the PHN covariance matrix $\boldsymbol{\Phi}$ and the coarse $\mathrm{CFO} v_{c}$, the covariance matrix $\boldsymbol{\Sigma}_{\boldsymbol{h}, v}$ can be approximated as $\boldsymbol{\Sigma}_{\boldsymbol{h}, v} \approx \boldsymbol{\Sigma}_{\hat{\boldsymbol{h}}, v_{c}}$.

The likelihood function of (11) can be given by

$$
\begin{aligned}
& \Lambda\left(\boldsymbol{r} ; v_{c}, v_{e}\right)=\frac{1}{(\pi)^{N} \operatorname{det}\left(\boldsymbol{\Sigma}_{\hat{\boldsymbol{h}}, v_{c}}\right)} \\
& \quad \times \exp \left\{-\left(\boldsymbol{r}-\boldsymbol{\Omega}_{v_{e}} \boldsymbol{\Omega}_{v_{c}} \boldsymbol{S} \hat{\boldsymbol{h}}\right)^{H}\left(\boldsymbol{\Sigma}_{\hat{\boldsymbol{h}}, v_{c}}\right)^{-1}\left(\boldsymbol{r}-\boldsymbol{\Omega}_{v_{e}} \boldsymbol{\Omega}_{v_{c}} \boldsymbol{S} \hat{\boldsymbol{h}}\right)\right\}
\end{aligned}
$$

where $v_{e}$ denotes the frequency offset estimation error $v-v_{c}$. Then, the frequency offset error estimate $\hat{v}_{e}$ can be obtained by

$$
\hat{v}_{e}=\min _{v_{e}}\left(\boldsymbol{r}-\boldsymbol{\Omega}_{v_{e}} \boldsymbol{\Omega}_{v_{c}} \boldsymbol{S} \hat{\boldsymbol{h}}\right)^{H}\left(\boldsymbol{\Sigma}_{\hat{\boldsymbol{h}}, v_{c}}\right)^{-1}\left(\boldsymbol{r}-\boldsymbol{\Omega}_{v_{e}} \boldsymbol{\Omega}_{v_{c}} \boldsymbol{S} \hat{\boldsymbol{h}}\right) .
$$

Using $\hat{\boldsymbol{B}}=\boldsymbol{S} \hat{\boldsymbol{h}}=[\hat{B}(0) \hat{B}(1) \cdots \hat{B}(N-1)]^{T}$, (14) can be calculated as

$$
\begin{aligned}
\hat{v}_{e}= & \arg \min _{v_{e}}\left\{-\boldsymbol{R}\left\{\boldsymbol{r}^{H}\left(\boldsymbol{\Sigma}_{\hat{\boldsymbol{h}}, v_{c}}\right)^{-1} \boldsymbol{\Omega}_{v_{e}} \boldsymbol{\Omega}_{v_{c}} \hat{\boldsymbol{B}}\right\}\right. \\
& \left.+\frac{1}{2}\left(\boldsymbol{\Omega}_{v_{e}} \boldsymbol{\Omega}_{v_{c}} \hat{\boldsymbol{B}}\right)^{H}\left(\boldsymbol{\Sigma}_{\hat{\boldsymbol{h}}, v_{c}}\right)^{-1}\left(\boldsymbol{\Omega}_{v_{e}} \boldsymbol{\Omega}_{v_{c}} \hat{\boldsymbol{B}}\right)\right\}
\end{aligned}
$$

where $\mathfrak{R}(x)$ is the real value of complex variable $x$. However, it is too complicate to obtain an exact solution of $\hat{v}_{e}$ in (15) [13]. Therefore, we assume that $v_{e}$ is sufficiently small to approximate $\exp \left(j \frac{2 \pi v_{e} n}{N}\right)$ by Taylor series expansion to the second order term;

$$
\exp \left(j \frac{2 \pi v_{e} n}{N}\right) \approx 1+j \frac{2 \pi v_{e} n}{N}-\frac{1}{2}\left(\frac{2 \pi v_{e} n}{N}\right)^{2} .
$$

Then, (15) can be given in the quadratic form of $v_{e}$ shown as 


$$
\hat{v}_{e} \approx \arg \min _{v_{e}}\left\{A v_{e}^{2}+B v_{e}+C\right\}
$$

and the estimate $\hat{v}_{e}$ is obtained by

$$
\hat{v}_{e}=-\frac{B}{2 A} \text {. }
$$

In (17) and (18), $A$ and $B$ are defined as follows:

$$
\begin{aligned}
A & =\frac{2 \pi^{2}}{N^{2}} \sum_{l=0}^{N-1} \sum_{n=0}^{N-1}\left\{n^{2} \mathfrak{R}\left\{r^{*}(l) \Sigma_{l, n}^{-1} \Omega_{v_{c}}(n) \hat{B}(n)\right\}\right\} \\
& -\frac{\pi^{2}}{N^{2}} \sum_{l=0}^{N-1} \sum_{n=0}^{N-1}\left\{(n-l)^{2} \Omega_{v_{c}}(n) \Omega_{v_{c}}^{*}(l) \Sigma_{l, n}^{-1} \hat{B}^{*}(l) \hat{B}(n)\right\} \\
B & =\frac{2 \pi}{N} \sum_{l=0}^{N-1} \sum_{n=0}^{N-1}\left\{n \mathfrak{I}\left\{r^{*}(l) \Sigma_{l, n}^{-1} \Omega_{v_{c}}(n) \hat{B}(n)\right\}\right\} \\
& +j \frac{\pi}{N} \sum_{l=0}^{N-1} \sum_{n=0}^{N-1}\left\{(n-l) \Omega_{v_{c}}(n) \Omega_{v_{c}}^{*}(l) \Sigma_{l, n}^{-1} \hat{B}^{*}(l) \hat{B}(n)\right\}
\end{aligned}
$$

where $\mathfrak{I}(x)$ is the imaginary value of complex variable $x$ and $\Sigma_{l, n}^{-1}$ is the $l$ th row and the $n$th column of $\left(\Sigma_{\hat{h}, v_{c}}\right)^{-1}$.

Assuming that PHN is negligible, the likelihood function of (11) can be given by

$$
\Lambda\left(\boldsymbol{r} ; v_{c}, v_{e}\right)=\frac{1}{\left(\pi \sigma_{w}^{2}\right)^{N}} \exp \left\{-\frac{1}{\sigma_{w}^{2}}\left\|\boldsymbol{r}-\boldsymbol{\Omega}_{v_{e}} \boldsymbol{\Omega}_{v_{c}} \hat{\boldsymbol{B}}\right\|^{2}\right\}
$$

where $\|\boldsymbol{x}\|^{2}=\boldsymbol{x}^{H} \boldsymbol{x}$. Using (16) and (21), the negligible-PHN CFO estimate (NPCE) $\hat{v}_{e}^{N P C E}$ is obtained by

$$
\hat{v}_{e}^{N P C E}=-\frac{N}{2 \pi} \cdot \frac{\sum_{n=0}^{N-1} n \mathfrak{J}\left\{r^{*}(n) \hat{B}(n) \Omega_{v_{c}}(n)\right\}}{\sum_{n=0}^{N-1} n^{2} \mathfrak{R}\left\{r^{*}(n) \hat{B}(n) \Omega_{v_{c}}(n)\right\}} .
$$

This NPCE algorithm, which ignores PHN, is easier to implement, as it does not require any knowledge of SNR.

\section{Simulation Results}

In our simulations, the DFT size, the CP size, the number of modulated sub-carriers and total system bandwidth are given as $N=64, N_{C P}=16, P=52$ and $B W=20 \mathrm{MHz}$, respectively. We consider a frequency selective fading channel whose sampled impulse response is given by $\boldsymbol{h}=[0.77+0.38 j, 0,0,0,0,0,0,0,0.58 j,-0.58-0.67 j]^{T}$ with $L=10$ [13]. Moreover, one frame is composed of $N_{F}$ OFDM symbols, the first symbol of each frame is set to be a preamble and the data sub-carriers are modulated by quadrature phase shift keying (QPSK). The PLL (phase locked loop) frequency synthesizer is considered for the PHN model [14]. The PHN has a standard deviation of $\phi_{R M S}$. It is generated using a single pole Butterworth filter of $3 \mathrm{~dB}$ bandwidth $100 \mathrm{kHz}$. It is assumed that the CFO

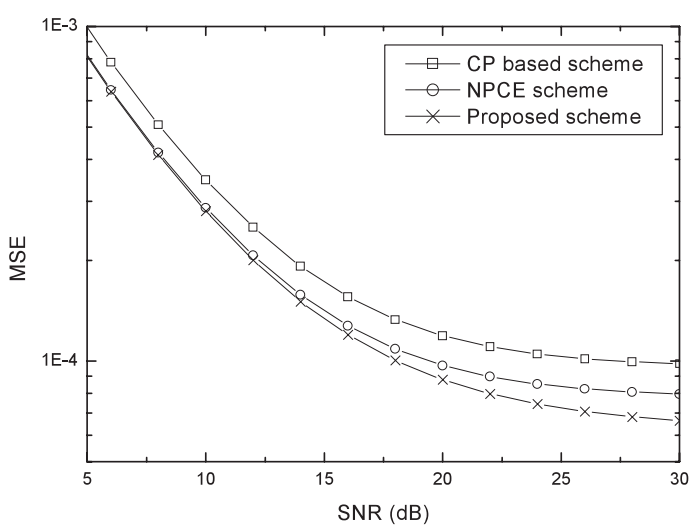

Fig. 2 Comparisons of MSE performance for CFO estimator with PHN standard deviation $\phi_{R M S}=2$ degree and $\mathrm{CFO} v=0.3$.

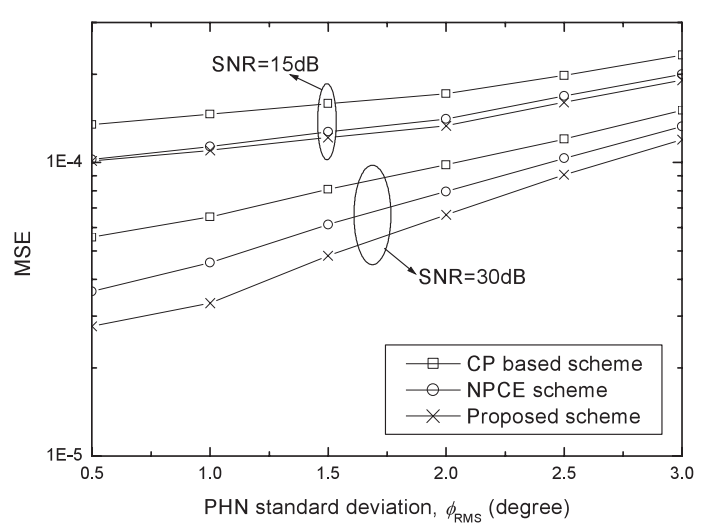

Fig. 3 Comparisons of MSE performance for CFO estimator according to the different values of PHN standard deviation at CFO $v=0.3$.

is constant in each frame. The estimation performance is evaluated by the mean square error (MSE) and the bit error rate (BER) performance. The MSE of the CFO estimator is defined as $E\left[\left|\hat{v}_{e}-v_{e}\right|^{2}\right]$.

In Fig. 2, we consider the MSE performance of the CFO estimator with the PHN standard deviation $\phi_{R M S}=2$ degree and CFO $v=0.3$. For $\mathrm{SNR} \geq 15 \mathrm{~dB}$, the MSE of the proposed CFO estimator is of the order of $10^{-4}$ or less. We can observe that the proposed scheme outperforms the $\mathrm{CP}$ based estimator [3] in all ranges of SNR and has a better performance compared with the NPCE scheme. It is because the proposed scheme uses the PHN covariance matrix $\Phi$ and SNR to obtain the CFO estimate. However, PHN and frequency offset estimation error cause an error floor of the proposed scheme. Figure 3 shows the MSE performance of the CFO estimator according to the different values of the PHN standard deviation $\phi_{R M S}$ at CFO $v=0.3$. It is seen that, for various PHN standard deviation conditions, the performance of the proposed scheme always outperforms the $\mathrm{CP}$ based scheme. Furthermore, the proposed scheme gives the better performance with positive gain than the MSE performance of the NPCE scheme at high SNR.

In Fig. 4, we present the BER performance of the CFO estimator with the PHN standard deviation $\phi_{R M S}=0.5 \mathrm{de}$ - 


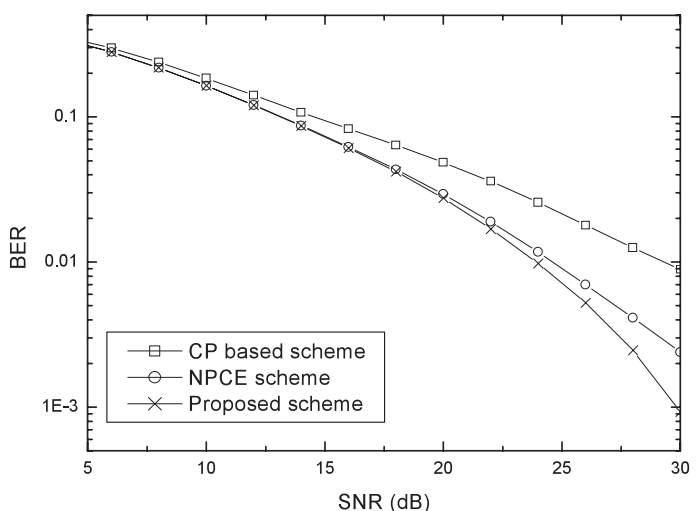

Fig. 4 Comparisons of BER performance for CFO estimator with PHN standard deviation $\phi_{R M S}=0.5$ degree, $\mathrm{CFO} v=0.3$ and frame size $N_{F}=$ 11.

gree, CFO $v=0.3$ and the frame size $N_{F}=11$. The performance of the proposed scheme is also compared with the performance of the NPCE scheme and the performance of the CP based scheme with the LS CIR estimation. It can be seen that the BER performance of the proposed scheme outperforms the BER performance of the CP based scheme with the LS CIR estimates. Moreover, the proposed scheme improves the BER performance for the SNR larger than $20 \mathrm{~dB}$ comparing with the NPCE scheme.

\section{Conclusion}

In this letter, we proposed a carrier frequency synchronization scheme for OFDM systems in the presence of PHN. The proposed scheme obtains performance improvement using CP-based CFO estimate, the LS channel estimate and the PHN covariance matrix. Simulation results have been shown that the proposed scheme has much better performance than the CP-based CFO estimation scheme. The proposed scheme presents better performance than the NPCE one for high SNR.

\section{Acknowledgments}

This work was supported partly by Brain Korea 21 Project in 2006 and partly by ITRC.

\section{References}

[1] M. Morelli and U. Mengali, "A comparison of pilot-aided channel estimation methods for OFDM systems," IEEE Signal Process., vol.49, no.12, pp.3065-3073, Dec. 2001.

[2] L. Deneire, P. Vandenameele, L. van der Perre, B. Gyselinckx, and M. Engels, "A low-complexity ML channel estimator for OFDM," IEEE Commun. Lett., vol.51, no.2, pp.135-140, Feb. 2003.

[3] J.J. van de Beek, M. Sandell, and P.O. Borjesson, "ML estimation of timing and frequency offset in OFDM systems," IEEE Signal Process., vol.45, no.7, pp.1800-1805, July 1997.

[4] T.M. Schmidl and D.C. Cox, "Robust frequency and timing synchronization for OFDM," IEEE Trans. Commun., vol.45, no.12, pp.1613-1621, Dec. 1997.

[5] A.J. Coulson, "Maximum likelihood synchronization for OFDM using a pilot symbol: Algorithms," IEEE J. Sel. Areas Commun., vol.19, no.12, pp.2486-2494, Dec. 2001.

[6] X. Ma, H. Kobayashi, and S.C. Schwartz, "Joint frequency offset and channel estimation for OFDM," Proc. Global Telecommunication Conf., vol.1, pp.15-19, Dec. 2003.

[7] T. Cui and C. Tellambura, "Robust joint frequency offset and channel estimation for OFDM systems," Proc. Vehicular Technology Conf., vol.1, pp.603-607, Los Angeles, CA, Sept. 2004.

[8] X. Wang, P. Ho, and Y. Wu, "Robust channel estimation and ISI cancellation for OFDM systems with suppressed features," IEEE J. Sel. Areas Commun., vol.23, no.5, pp.963-972, May 2005.

[9] A.G. Armada, "Understanding the effects of phase noise in orthogonal frequency division multiplexing," IEEE Trans. Broadcast., vol.47, no.5, pp.580-583, May 1998.

[10] L. Piazzo and P. Mandarini, "Analysis of phase noise effects in OFDM modems," IEEE Trans. Commun., vol.50, no.10, pp.16961705, Oct. 2002.

[11] H.G. Ryu and Y.S. Lee, "Phase noise analysis of the OFDM communication system by the standard frequency deviation," IEEE Trans. Consum. Electron., vol.49, no.1, pp.41-47, Feb. 2003.

[12] K. Nikitopoulos and A. Polydoros, "Phase-impairment effects and compensation algorithms for OFDM systems," IEEE Trans. Commun., vol.53, no.4, pp.698-707, April 2005.

[13] S. Attallah, "Blind estimation of residual carrier offset in OFDM systems,” IEEE Signal Process. Lett., vol.11, no.2, pp.216-219, Feb. 2004.

[14] IEEE P802.11 Task Group G, http://grouper.ieee.org/groups/802/11/ Reports/tgg_update.htm

[15] A. Mehrotra, "Noise analysis of phase-locked loops," IEEE Circuits Syst. Mag., vol.49, no.9, pp.1309-1316, Sept. 2002. 